\title{
Accelerated convergence method for fast Fourier transform simulation of coupled cavities
}

\author{
R. A. Day, ${ }^{1, *}$ G. Vajente, ${ }^{2,3}$ and M. Pichot du Mezeray ${ }^{4}$ \\ ${ }^{1}$ European Gravitational Observatory (EGO), I-56021 Cascina (PI), Italy \\ ${ }^{2} I N F N$, Sezione di Pisa, I-56127 (PI), Italy \\ ${ }^{3}$ Currently at LIGO Laboratory 100-36, California Institute of Technology, Pasadena, California 91125, USA \\ ${ }^{4}$ ARTEMIS/Observatoire de la Côte d'Azur, Nice, France \\ *Corresponding author: richard.day@ego-gw.it
}

Received December 10, 2013; revised January 17, 2014; accepted January 23, 2014; posted January 29, 2014 (Doc. ID 202831); published February 27, 2014

Fast Fourier transform (FFT) simulation was used to calculate the power and spatial distribution of resonant fields in optical cavities. This is an important tool when characterizing the effect of imperfect geometry and mirror aberrations. This method is, however, intrinsically slow when the cavities are of relatively high finesse. When this is the case, an accelerated convergence scheme may be used to calculate the steady-state cavity field with a speed that is orders of magnitude faster. The rate of convergence of this method, however, is unpredictable, as many different factors may detrimentally affect its performance. In addition, its use in multiple cavity configurations is not well understood. An in-depth study of the limitations and optimization of this method is presented, together with a formulation of its use in multiple cavity configurations. This work has not only resulted in consistent improvement in performance and stability of the accelerated convergence method but also allows the simulation of optical configurations, which would not previously have been possible. (c) 2014 Optical Society of America OCIS codes: (070.7345) Wave propagation; (070.5753) Resonators. http://dx.doi.org/10.1364/JOSAA.31.000652

\section{INTRODUCTION}

Optical simulation is an important tool for understanding and building optical cavities. The physics of optical cavities is well understood [1], and so an analytical approach can give many accurate predictions about the design and resulting performance of such a cavity. However, when designing coupled optical cavities or even a single optical cavity to extremely high requirements, it is necessary to take into account many subtleties such as cavity mirror roughness, alignment, and realistic locking points. For such effects, an analytical approach becomes less practical and optical simulations come into play.

One popular approach is to use modal simulations [2,3]. Modal simulators expand the laser field into a base of Hermite-Gaussian or Laguerre-Gaussian modes for which the base is the eigenmode of the perfect cavity. These simulators have the advantage of being very fast when considering low spatial frequency aberrations in the cavity. However, the simulation of higher spatial frequency aberrations requires a number of higher order modes (HOMs) that increase as $\rho^{4}$ (where $\rho$ is the spatial frequency) [4], which in turn dramatically increases the simulation time.

An alternative approach is the so-called fast Fourier transform (FFT) simulation [5], or angular spectrum propagation method. The FFT simulations expand the laser field into plane wave components with different transverse frequency components by means of a FFT. The plane waves are then propagated independently and the resulting field is computed by inverse Fourier transform where it can then be transmitted through or reflected off an optical component before being propagated again. In simple optical configurations such as a Michelson interferometer, a finite number of operations are needed to propagate the input field to the output ports. However, in an optical cavity the fields propagate an infinite number of times inside the system, and the steady-state solution is given by the interference of all the round trips. In this case, the propagations, transmissions, and reflections of the intra-cavity field may be iterated until it has converged to its steady state. The FFT simulation is much faster than modal simulation when there are high spatial frequency aberrations in the cavity. With such simulators, it is possible to simulate highly degenerate cavities and even round-trip losses due to mirror clipping or scattered light.

The FFT simulator has proved to be an extremely important tool for many applications such as the design of gravitational wave (GW) interferometers [6]. These experiments, which are composed of high-finesse kilometer-scale coupled cavities, demand a perfect understanding of the mechanisms involved in the use of imperfect cavity mirrors. It is the use of FFT simulators that has allowed researchers to define the extremely high polishing requirements for the core optics of GW interferometers.

The increasing complexity of applications such as GW interferometers has highlighted limitations in FFT simulation tools. The FFT simulation, which uses standard convergence, replicates the light circulating inside the cavity. Therefore, the time taken (or number of iterations) for the intra-cavity power to buildup to steady state increases with the finesse of the cavity. This fundamental issue was addressed by Saha [7] with the proposition of an accelerated convergence scheme. This method basically treats the convergence as a matrix inversion problem and demonstrates how a traditional over-relaxation method may be used to arrive at steady state in as few 
iterations as possible. This work was an important contribution to the field, and it is currently implemented in most FFT simulators in the GW community.

The accelerated convergence scheme works extremely well for near perfect stable cavities. However, it has been found that the performance of this method can be degraded by a large number of factors dependent on both the optical configuration (e.g., cavity geometry, operating points, and beam matching) and the numerical implementation (e.g., aliasing and machine precision). The rate of convergence of this method is therefore relatively unpredictable in a general sense to the extent that, in some cases, it can take longer than the traditional convergence method.

The second issue with this scheme is that its use with coupled cavities is not straightforward. Attempts to accelerate two cavities that are coupled usually result in large instabilities that prevent convergence to the steady state. One solution to this problem is to accelerate the cavity containing high power and use traditional convergence on the one containing lower power. However, this solution may only be applied for a few specific optical configurations. A "global relaxation" scheme was proposed in [으, 9$]$ whereby steady-state fields for each cavity are determined simultaneously in order to minimize a specially weighted sum of the iteration errors for all relaxed fields. However, this system is also reported to have instabilities that are heavily configuration dependent.

In this paper, we address these issues concerning the accelerated convergence scheme. We annotate this discussion by giving example results of simulations that have been developed in MATLAB [10]. In Section 2, we will summarize the state-of-the-art FFT simulation for a single cavity. We will also describe our criteria for determining when steady state has been reached, which will be used extensively throughout this article. In Section 3, we will discuss how different optical configurations can degrade the performance of the accelerated convergence and what measures may be taken in order to optimize the convergence using such configurations. Finally, in Section $\underline{4}$, we will propose an accelerated convergence scheme for multiple coupled cavities and give some examples of where its use will be of great benefit. This work lays the foundation for using accelerated convergence in any arbitrary optical configuration.

\section{ACCELERATED CONVERGENCE OF A SINGLE CAVITY}

In this section, we will discuss the basic principles needed to simulate a single optical cavity using FFT simulation with the accelerated convergence scheme.

\section{A. Simulation Operators}

Any FFT simulation consists of a series of propagations, transmissions, and reflections. Figure 1 shows a schematic of a field in plane A propagated to plane $B$ where it is then reflected off and transmitted through a "thin" mirror having amplitude reflectivity and transmission of $r$ and $t$, respectively. Given the field in plane $\mathrm{A}, E_{A}(x, y)$ as a function of the transverse coordinates, the field in plane $\mathrm{B}$ is given by:

$$
E_{B}(x, y)=\mathcal{P}\left[E_{A}(x, y)\right]
$$

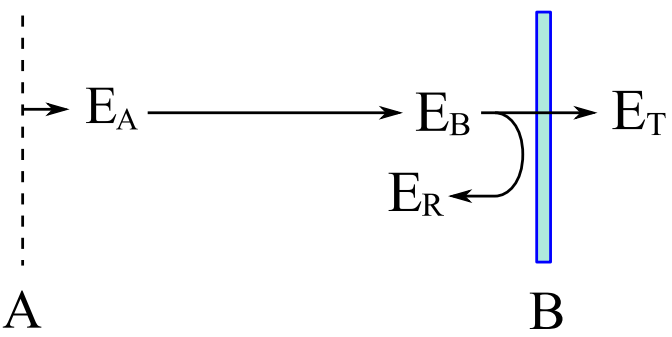

Fig. 1. Definition of field propagated to, reflected off, and transmitted through a thin mirror.

Here, $\mathcal{P}$ is the propagation operator, which is the solution of the paraxial diffraction Eq. (1):

$$
E_{B}(x, y)=\mathcal{F}^{-1}\left[M\left(k_{x}, k_{y}\right) \cdot \mathcal{F}\left[E_{A}(x, y)\right]\right]
$$

where $\mathcal{F}\left[E_{A}(x, y)\right]$ is the bi-dimensional Fourier transform of the field, which returns a function of the spatial frequency coordinates $k_{x}$ and $k_{y}$; and $\mathcal{F}^{-1}$ is the inverse Fourier transform, which returns a function of the spatial coordinates. $M\left(k_{x}, k_{y}\right)$ is the propagation kernel given by:

$$
M\left(k_{x}, k_{y}\right)=\exp \left[-i k L+i \frac{k_{x}^{2}+k_{y}^{2}}{2 k} L\right]
$$

where $k$ is the wavenumber and $L$ is the propagation distance. This expression is accurate within the paraxial approximation.

The field $E_{B}$ will then be reflected and transmitted at the mirror to give the fields:

$$
\begin{aligned}
& E_{R}(x, y)=\mathcal{R}_{B}\left[E_{B}(x, y)\right], \\
& E_{T}(x, y)=\mathcal{T}_{B}\left[E_{B}(x, y)\right] .
\end{aligned}
$$

Here, $\mathcal{R}_{B}$ and $\mathcal{T}_{B}$ are the reflection and transmission operators, respectively, and correspond to the operations:

$$
E_{R}(-x, y)=i r D(x, y) \exp [2 i k H(x, y)] E_{B}(x, y),
$$

$$
E_{T}(x, y)=t D(x, y) \exp [-i k G(x, y)] E_{B}(x, y)
$$

where $H(x, y)$ is the height map (in $z$ ) of the reflecting surface and $G(x, y)$ is the optical path length map (in z) in transmission. $D(x, y)$ represents the finite aperture of the mirror, taking values of 1 for all points inside the mirror and 0 outside. Note that in the computation of the reflected field, it is necessary to invert the sign of one of the coordinates to conserve the right hand coordinate system. In this definition, we have used the convention that all reflections are multiplied by $i$, although other literature may use a different convention.

In the actual numerical implementation of the above equations, a discrete sampling of the fields over a window of fixed size is used, with $N$ samples per direction. Each field is then described by a $N \times N$ matrix, as well as the functions $G, H$, and $D$. The continuous Fourier transform is finally substituted with the discrete FFT. The propagation kernel is also described by a $N \times N$ matrix that is sampled in the Fourier space. 


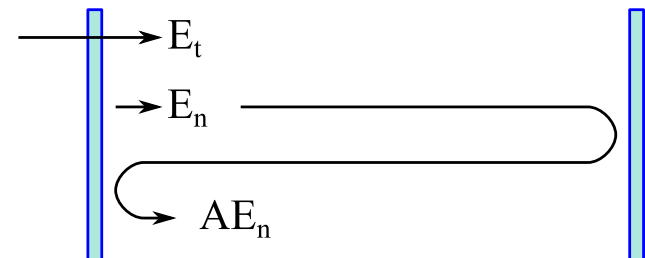

Fig. 2. Definition of field and operators in the simulation of a single Fabry-Perot cavity.

\section{B. Convergence Scheme}

The accelerated convergence scheme for a single cavity was first described in [7]. Figure 2 shows a schematic diagram of the single cavity setup. $E_{n}$, referred to as the guess field, is the estimation of the cavity field at a given iteration, while the pump field, after transmission through the input mirror, is called $E_{t}$. The simple iterative approach to finding the steady-state solution is to compute one round trip of the field:

$$
E_{n+1}^{\prime}=E_{t}+\mathcal{A}\left[E_{n}\right]
$$

where $\mathcal{A}=\mathcal{R}_{A} \mathcal{P} \mathcal{R}_{B} \mathcal{P}$ is the operator describing the round-trip propagation inside the cavity. The acceleration proposed in [7] is to construct a new guess field as a linear combination

$$
E_{n+1}=a_{n} E_{n}+b_{n} E_{n+1}^{\prime}
$$

with coefficients $a_{n}$ and $b_{n}$ to be determined at each iteration. The error we make in using this new guess field can be estimated as the amount of change in the field over an entire round-trip propagation:

$$
\Delta_{n+1}=E_{n+1}-\left(E_{t}+\mathcal{A}\left[E_{n+1}\right]\right) .
$$

If we were able to find the exact solution, this change would be zero. This error is a function of the $a_{n}$ and $b_{n}$ coefficients, which can be found by substituting Eq. (ㅁ) into Eq. (10):

$$
\Delta_{n+1}=a_{n}\left(E_{n}-\mathcal{A}\left[E_{n}\right]\right)+b_{n}\left(E_{n+1}^{\prime}-\mathcal{A}\left[E_{n+1}^{\prime}\right]\right)-E_{t} .
$$

Introducing the following definitions

$$
\begin{aligned}
\mathbf{D} & =\left[\begin{array}{c}
E_{n}-\mathcal{A}\left[E_{n}\right] \\
E_{n+1}^{\prime}-\mathcal{A}\left[E_{n+1}^{\prime}\right]
\end{array}\right]^{T} \\
\mathbf{X}_{A} & =\left[\begin{array}{l}
a_{n} \\
b_{n}
\end{array}\right],
\end{aligned}
$$

where $\mathbf{x}^{T}$ indicates the matrix transpose, the error can be written in a more compact form

$$
\Delta_{n+1}=\mathbf{D X}_{A}-E_{t}
$$

The best choice of the coefficients is the one that minimizes the squared value of the error:

$$
e=\Delta_{n+1}^{*} \Delta_{n+1}=\sum\left(\mathbf{X}_{A}^{+} \mathbf{D}^{+}-E_{t}^{*}\right)\left(\mathbf{D} \mathbf{X}_{A}-E_{t}\right)
$$

where the summation is over all the samples of the window used and $\mathbf{x}^{+}$indicates the Hermitian transpose. The minimum is found through requiring the derivative of the above equation with respect to $\mathbf{X}_{A}^{+}$to be zero:

$$
\left(\sum \mathbf{D}^{+} \mathbf{D}\right) \mathbf{x}_{A}=\sum \mathbf{D}^{+} E_{t}
$$

which corresponds to the system of equations of [7]. We may therefore determine the coefficients $a_{n}$ and $b_{n}$ by solving numerically

$$
\mathbf{X}_{A}=\left(\sum \mathbf{D}^{+} \mathbf{D}\right)^{-1}\left(\sum \mathbf{D}^{+} E_{t}\right)
$$

\section{Convergence Figure-of-Merit}

An important aspect of the convergence algorithm is to determine when the guess field has reached steady state. It is therefore necessary to define a figure-of-merit that gives this information. This figure-of-merit will then be compared with a user defined threshold in order to decide if steady state has been reached. The choice of threshold will be a compromise between the required simulation speed and required accuracy. A natural choice of figure-of-merit for this kind of simulation is the fractional error in power between the field after $n$ round trips and $n+1$ round trips yielding:

$$
\delta^{\prime}=\frac{\left|\sum E_{n}^{*} E_{n}-\sum E_{n+1}^{*} E_{n+1}\right|}{\sum E_{n+1}^{*} E_{n+1}} .
$$

However, by inspection, we can see that this type of figureof-merit will not take into account errors in phase between successive iterations, nor will it take into account errors in the transverse distribution of power. With this in mind, a preferable figure-of-merit would take the form of:

$$
\delta=2 \sqrt{\frac{\sum\left(E_{n}^{*}-E_{n+1}^{*}\right)\left(E_{n}-E_{n+1}\right)}{\sum E_{n+1}^{*} E_{n+1}}} .
$$

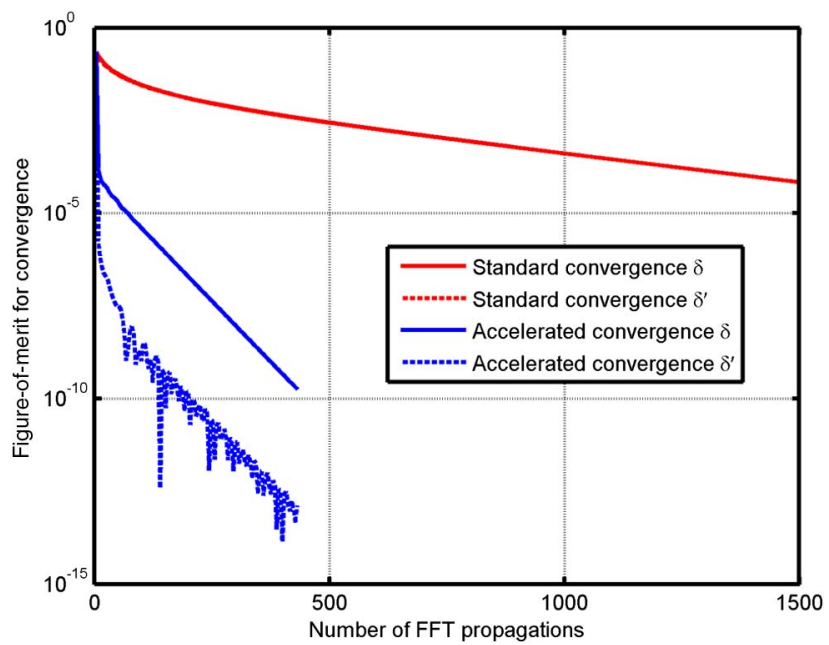

Fig. 3. Comparison of figure-of-merit $\delta$ and $\delta^{\prime}$ for standard and accelerated convergence. $\delta$ and $\delta^{\prime}$ for standard convergence are superposed. Concave-concave cavity of length $3000 \mathrm{~m}$ was used for this case, with a radius of curvature for the input and end mirror being 1420 and $1683 \mathrm{~m}$, respectively. Transmission values of input and end mirrors were $1.4 \%$ and $1 \mathrm{ppm}$, respectively. Mirror diameters were $350 \mathrm{~mm}$. Input beam was mode-matched to the cavity. 
In Fig. 3, we compare the evolution of these two figures-ofmerit as the cavity field converges. In the $x$ axis, we prefer to count the number of FFT propagations rather than the number of iterations used in other published works. The reason for this choice is the introduction of "smoothed" and "averaged" acceleration in Section 3.B, where the time to calculate one iteration greatly depends on the smoothing and averaging number chosen. Counting the number of FFT propagations is therefore a better indicator of convergence time when comparing different convergence methods and parameters. We see that for standard convergence the two figures-of-merit are equivalent. However, for accelerated convergence $\delta^{\prime}$, the fractional error in power has a relatively erratic descent and prematurely indicates that steady state has been achieved. This generates some doubt as to the validity of the state of convergence. The preferred figure-of-merit $\delta$, however, exhibits a smooth descent. The shape and rate of descent of this figure-of-merit will be a useful visual guide for the optimization of the convergence throughout this paper.

\section{Resonance Length}

Finding the resonance length or "locking point" for the cavity is another important aspect to consider in order to faithfully reproduce the physical system. The simulator should normally use the same type of error signals that are used experimentally. For example, for a cavity that is locked using the Pound-Drever-Hall technique [11], it would be necessary to generate an error signal using also the upper and lower sidebands of the phase modulated laser. The simulation of additional fields poses no fundamental difference with respect to the carrier field. It is only necessary to modify the propagator of Eq. (3) to include the additional phase shift due to the frequency difference of the sideband with respect to the carrier.

However, the simulation of a Pound-Drever-Hall error signal is outside the scope of this paper and we will restrict our discussion to a "coarse" lock of the cavity. We base this lock on the principle that the resonant cavity field should undergo a round-trip phase change that is an integer multiple of $2 \pi$. We therefore compared the phase of the cavity field and the same field after one round trip. We then modified the phase of the propagation matrix accordingly, such as to modify the microscopic length of the cavity. For a linear Fabry-Perot cavity this yields

$$
\begin{gathered}
\phi=\operatorname{Arg}\left(\sum\left(\mathcal{A}\left[E_{n}\right]\right)^{*} E_{n}\right), \\
M^{\prime}\left(k_{x}, k_{y}\right)=e^{-\phi / 2} M\left(k_{x}, k_{y}\right) .
\end{gathered}
$$

Here, Arg is a function that returns the phase of a complex number. The factor $1 / 2$ in the phase corresponds to the fact that the matrix propagator, $M$, is used twice to achieve the round trip of the field. In different optical configurations this factor might vary; for example, for a triangular cavity when each space propagator is used once, one can add the phase to only one propagator or divide it among all of them.

It would be inefficient to wait for convergence of the field before applying this length change. We prefer to apply the length change after each iteration such as to progressively approach the locking point as the cavity field converges to steady state. In Fig. 4, we compare the convergence of the

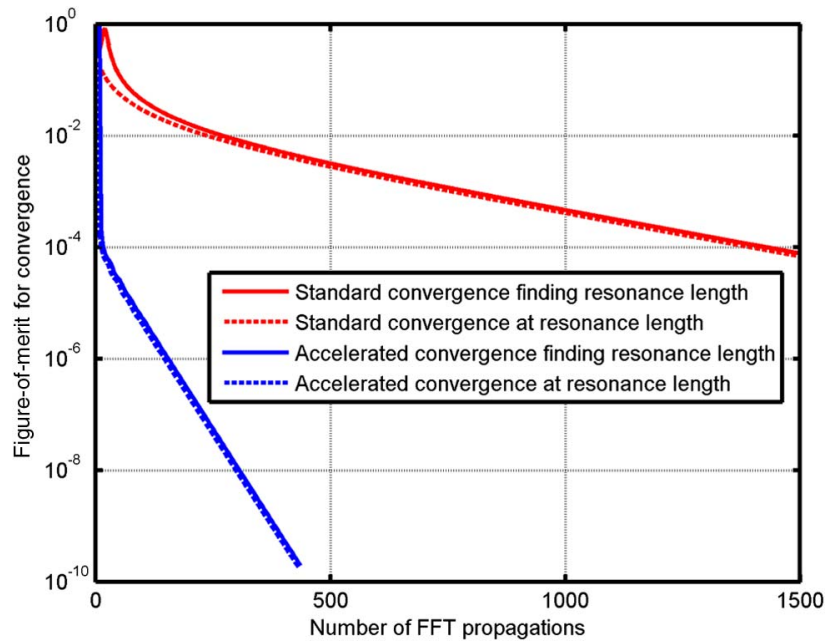

Fig. 4. Comparison of convergence with two different algorithms. Solid lines: when starting with an arbitrary microscopic cavity length and converging the field while modifying the phase of $\mathrm{M}$ after each iteration. Dashed lines: when starting with the resonant microscopic length and converging without modifying the phase of $M$.

cavity field for two cases. Specifically, these cases included (1) starting with an arbitrary microscopic cavity length and converging the field while modifying the phase of $M$ after each iteration, and (2) starting with the resonant microscopic length and converging without modifying the phase of $M$. We found that very little time was lost determining the coarse lock during convergence of the field. An extension of this work would be to then use "real" locking signals, once the coarse lock has been found, in order to fine tune the locking point.

\section{UNDERSTANDING AND IMPROVING PERFORMANCE}

As we saw in the previous section, the accelerated convergence scheme is capable of dramatically increasing the convergence speed of a high finesse optical cavity. However, when using this scheme in an arbitrary configuration with realistic mirror aberrations, a number of technical difficulties are encountered. In this section, we will discuss some of these issues and, where possible, we propose how to best deal with them in order to optimize the convergence speed. These suggestions not only apply for a single cavity but also for the case of multiple coupled cavities that will be presented in the next section.

\section{A. Limits of Working Precision}

The numerical calculation of the guess field requires solving Eq. (16). This necessitates finding the inverse or pseudoinverse of the term $\sum \mathbf{D}^{+} \mathbf{D}$. However, as the convergence approaches steady state this matrix becomes more and more singular. The calculated guess fields, and hence the convergence, can therefore become unstable before the required accuracy has been achieved. This problem is accentuated when accelerated convergence is considered for multiple coupled cavities where there are potentially large differences in power. The nature of this instability will depend on how the inverse is calculated. In Fig. 5 , we compare the use of different numerical methods for solving Eq. (16). 
The default working precision for the simulations is double precision. A direct inverse of the matrix results in an uncontrollable instability. This problem is addressed using a singular value decomposition (SVD) method. The SVD method is a standard computation that decomposes the matrix into three matrices such that

$$
\sum \mathbf{D}^{+} \mathbf{D}=\mathbf{U S V}^{+}
$$

Here, $S$ is a diagonal matrix with nonnegative diagonal elements in decreasing order and $U$ and $V$ are unitary matrices. In order to filter out singular values, elements on the diagonal of $S$ whose ratio with the maximum value is inferior to a certain threshold are set to zero. An element-wise reciprocal is then made of all remaining nonzero values. The resulting matrix $S_{\text {rec }}$ is used to determine the inverse matrix

$$
\left(\sum \mathbf{D}^{+} \mathbf{D}\right)^{-1}=\mathbf{V S}_{\mathrm{rec}}^{\prime} \mathbf{U}^{+}
$$

We can see in Fig. $\underline{5}$ that by choosing an appropriate threshold the instability is avoided. However, the system continues to converge at a much slower rate. There are many other methods for finding the pseudo-inverse of this matrix, however, to the best of the authors' knowledge, the working precision will continue to hinder the convergence speed or stability below a certain value for the figure-of-merit.

The easiest and most effective solution to this bottleneck is to double the working precision for this part of the calculation. We therefore calculate Eq. (16) and only Eq. (16) in quadruple precision. We used a third party toolbox [12] to provide this functionality in MATLAB. Because the FFT and inverse FFT calculations were the most time consuming operations in the simulation, the implementation of quadruple precision for Eq. (16) had a negligible effect on the time taken for one iteration. In the third plot of Fig. 5, we can see that the doubling of precision (using quadruple precision in this case) for this part of the calculation results in the rate of convergence remaining stable down to the required value for the figureof-merit.

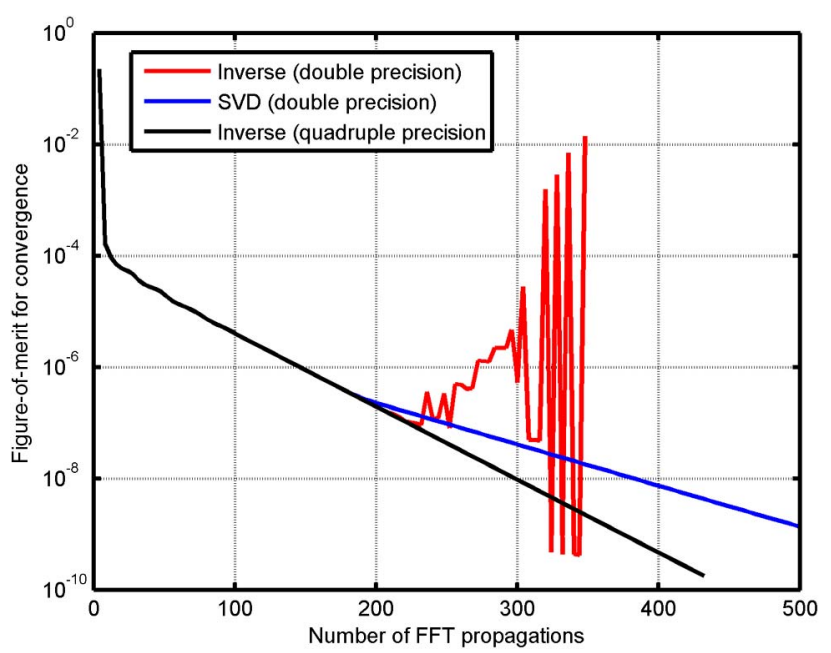

Fig. 5. Comparison of three numerical configurations for calculating Eq. (16).

\section{B. Cavities with Large HOM Content}

In order to measure the usefulness of accelerated convergence in a general case we must consider its use in different optical configurations with various kinds of imperfections. The empirical conclusion of this study is that a high proportion of HOMs in the cavity slows down convergence. This can be caused by various factors such as high degeneracy of the cavity, resonance condition, beam mismatching, mirror aberrations, and large apertures. Figure 6 shows how the convergence is modified by the presence of HOMs in the cavity. The solid red curve shows the accelerated convergence, as before, of an ideal cavity. The remaining red, blue, and green curves show the convergence of the same cavity for which the mirrors have a random roughness with varying rms. These maps will scatter light into HOMs that could be on or near resonance. We can see that, at first, the cavity converges in the same way. However, at a certain point (depending on the mirror rms) the rate of convergence suddenly reduces. This effect is interpreted as the error in the HOM convergence becoming dominant. As the steady-state solution for a large number of modes has many more degrees of freedom, it takes longer to converge. It should be noted that the standard convergence (black curves) was totally unaffected by the presence of HOMs. The authors believe that this reduction in convergence rate due to HOMs is an intrinsic limitation of the presented accelerated convergence scheme.

However, we identified other possibilities that we may consider. To further improve the convergence rate of the accelerated convergence method described above, we found empirically that it is useful to extend the number of fields used in the guesses. For example, in the case of a simple cavity we can then write

$$
E_{n+1}=a_{n} E_{n}+b_{n} E_{n+1}^{\prime}+c_{n} E_{n+2}^{\prime}+\cdots+d_{n} E_{n+N}^{\prime} .
$$

This change is easily inserted into the equations described in the previous sections by simply allowing the vectors defined in Eqs. (12) and (35) to accommodate more elements. We refer to this method as "smoothed acceleration."

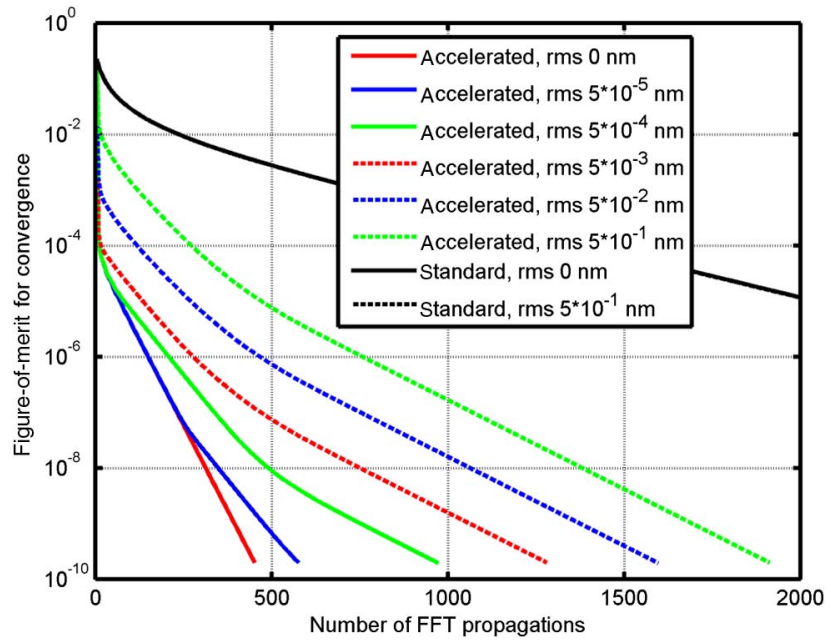

Fig. 6. Comparison of convergence when realistic mirror maps, with varying rms, are added to cavity mirrors. Plots for standard convergence case are superposed. 
A second improvement in the convergence rate can be obtained if the simple fields $E_{j}^{\prime}$ used in the estimates are substituted with averages over multiple round trips:

$$
\left\langle E_{n}^{\prime}\right\rangle_{M}=\frac{1}{M} \sum_{j=0}^{M-1} E_{n+j}^{\prime}
$$

Clearly, Eq. (23) must be modified accordingly to avoid overlapping averages:

$$
E_{n+1}=a_{n}\left\langle E_{n}^{\prime}\right\rangle_{M}+b_{n}\left\langle E_{n+M}^{\prime}\right\rangle_{M}+\cdots+c_{n}\left\langle E_{n+N M}^{\prime}\right\rangle_{M}
$$

We refer to this technique as "averaged acceleration." When both smoothed and averaged acceleration is used, the number of FFT propagations per iteration is simply given by $N M$.

In Fig. 7, we show how the smoothed and averaged acceleration can improve the convergence rate. We can see that, in this example, the convergence rate may be improved by up to a factor of five both for ideal cavities and cavities with realistic mirrors. The smoothed acceleration method was more effective than the averaged acceleration method. However, for the former all the fields must be kept in the computer memory whereas as for the latter it is sufficient to progressively sum the fields. Keeping the computer memory limitation in mind, it is often convenient to use a combination of both methods in one simulation.

\section{Problems of Aliasing}

The angular spectrum beam propagation method that is used in this study has the intrinsic problem of aliasing. Light that reaches the side of the FFT window should normally disappear. However, the aliasing effect results in it reappearing on the opposite side of the window with the same direction of propagation. In normal FFT simulations this effect is undesirable, but it may be tolerated to a certain extent. However, in accelerated convergence, this aliasing can have catastrophic effects. This unwanted light resulting from aliasing is equivalent to HOMs and therefore greatly reduces the convergence

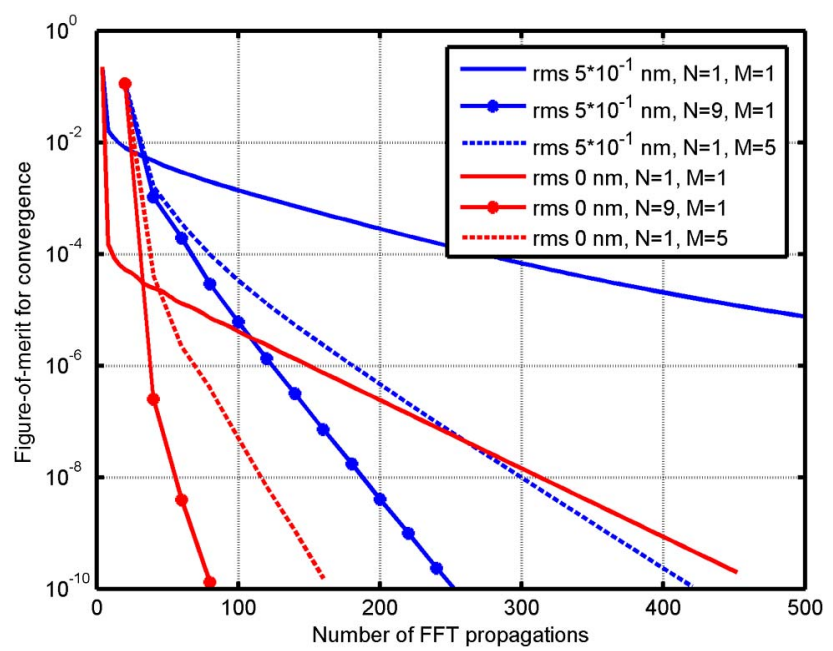

Fig. 7. Improvement in accelerated convergence by using smoothed and averaged acceleration in the case of perfect mirrors (red) and realistic mirrors with $\mathrm{rms} 5 \times 10^{-1} \mathrm{~nm}$. In the case of $\mathrm{N}=1, \mathrm{M}=1$ corresponds to the standard accelerated convergence. Cases $\mathrm{N}=9, \mathrm{M}=1$ and $\mathrm{N}=1, \mathrm{M}=5$ carry out the same number of propagations per iteration.

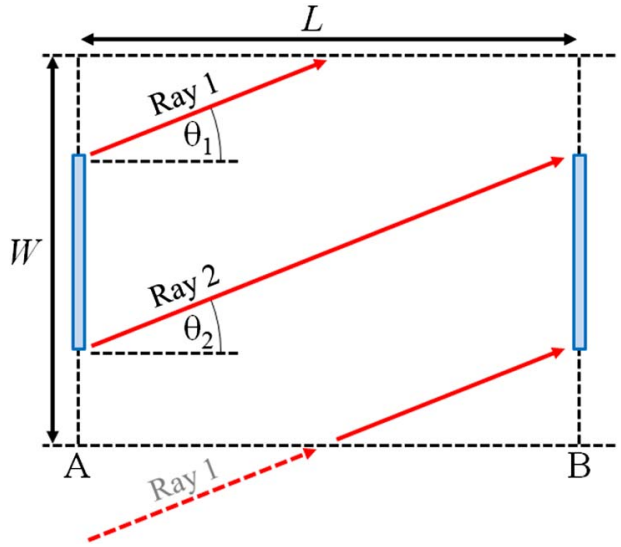

Fig. 8. Schematic diagram showing limiting condition for onset of FFT aliasing.

speed. As this effect is totally nonphysical, we wish to completely eliminate this problem. We will use an approach similar to that described in $[\underline{9}, \underline{13}]$. In Fig. $\underline{8}$, we show a schematic diagram of light scattered at large angles off mirror $\mathrm{A}$ and propagating to mirror B. Ray 1 shows the limiting case of the aliased scattered light hitting the edge of mirror $\mathrm{B}$. We therefore wish to eliminate light scattering at angles greater than $\theta_{1}$. Ray 2 shows the limiting case of light scattered off the bottom of mirror A and hitting the top of mirror B. We therefore wish to keep light scattering at angles smaller than $\theta_{2}$. If we choose the FFT window, $W$, to be exactly two times larger than the mirrors, then $\theta_{c}=\theta_{1}=\theta_{2}$. Using the small angle approximation we may write

$$
\theta_{c} \simeq \frac{W}{2 L}
$$

The angle at which light is scattered is related to the spatial frequency, $\rho$, of the mirror structure by

$$
\theta \simeq \lambda \rho
$$

This again uses the small angle approximation. Therefore, the spatial frequency cutoff to avoid aliasing is given by

$$
\rho_{c}=\frac{W}{2 L \lambda}
$$

This filtering is implemented after building the propagation kernel, $M$, in Eq. (3). All points for which $\left(k_{x}^{2}+k_{y}^{2}\right)>\rho_{c}^{2}$ are set to zero. Figure 9 gives an example of convergence with and without the filtering. We can see that with no filtering even a cavity with ideal mirrors can result in the characteristic sudden reduction in rate of convergence; for nonideal mirrors, the effect was worse. After implementation of the filtering, however, we found that we were no longer susceptible to the increased convergence time due to aliasing.

\section{DOUBLE AND MULTIPLE CAVITIES}

In this section, we will extend the accelerated convergence scheme for a single cavity to that of any arbitrary combination of coupled cavities.

The extension to an arbitrary number of cavity and pump fields is more easily understood starting from a double cavity 


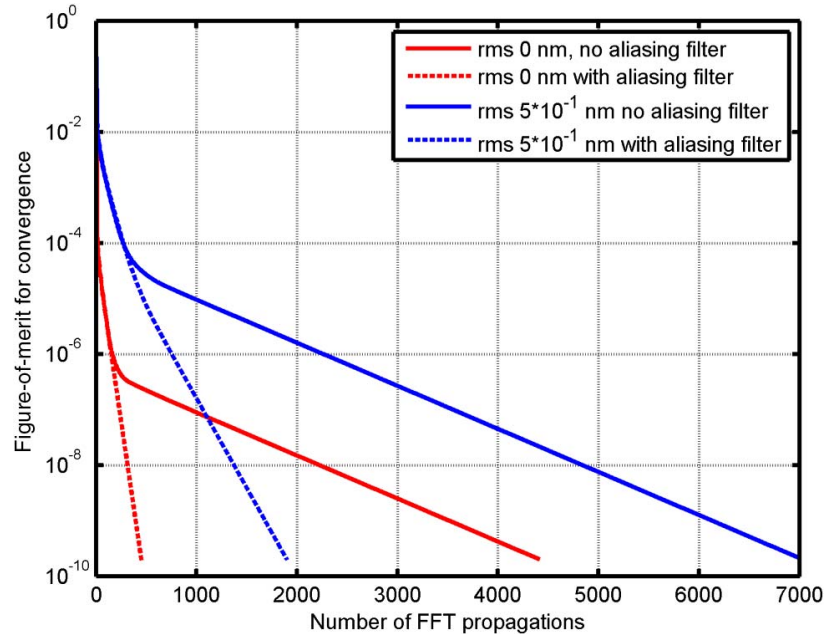

Fig. 9. Comparison of convergence with (dashed lines) and without (solid lines) aliasing filter using perfect cavity mirrors (red curves) and mirror maps having rms of $5 \times 10^{-1}$ (blue curves).

example, like the one shown in Fig. 10. For the sake of generality, we considered also the possibility of a pump field entering directly into the second cavity. This will allow us to generalize to an arbitrary system.

The iterative relations to be used without any acceleration are:

$$
\begin{aligned}
& E_{A, n+1}^{\prime}=E_{A, t}+\mathcal{D}\left[E_{B, n}\right]+\mathcal{A}\left[E_{A, n}\right] \\
& E_{B, n+1}^{\prime}=E_{B, t}+\mathcal{B}\left[E_{B, n}\right]+\mathcal{C}\left[E_{A, n}\right] .
\end{aligned}
$$

Following the same approach used for the single cavity, we write the best guess for the fields at the next iteration as linear combinations:

$$
\begin{aligned}
& E_{A, n+1}=a E_{A, n}+b E_{A, n+1}^{\prime} \\
& E_{B, n+1}=c E_{B, n}+d E_{B, n+1}^{\prime} .
\end{aligned}
$$

The error we make using these estimations is given, as before, by the change of this field when undergoing a full round-trip propagation:

$$
\begin{aligned}
& \Delta_{A, n+1}=E_{A, n+1}-\left[E_{A, t}+\mathcal{D}\left[E_{B, n+1}\right]+\mathcal{A}\left[E_{A, n+1}\right]\right] \\
& \Delta_{B, n+1}=E_{B, n+1}-\left[E_{B, t}+\mathcal{B}\left[E_{B, n+1}\right]+\mathcal{C}\left[E_{A, n+1}\right]\right] .
\end{aligned}
$$

To simplify to some extent the notation, we introduce the following definitions:

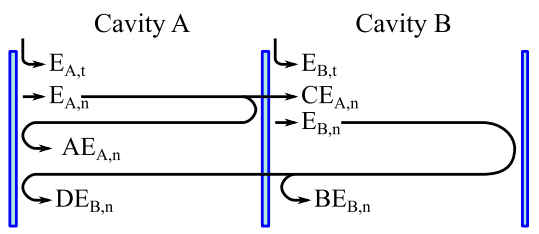

Fig. 10. Definition of field and operators in the simulation of a double Fabry-Perot cavity.

$$
\begin{aligned}
\mathbf{X}_{A} & =\left(\begin{array}{l}
a \\
b
\end{array}\right) \\
\mathbf{X}_{B} & =\left(\begin{array}{l}
c \\
d
\end{array}\right) \\
\mathbf{F}_{A} & =\left(\begin{array}{c}
E_{A, n}-\mathcal{A}\left[E_{A, n}\right] \\
E_{A, n+1}^{\prime}-\mathcal{A}\left[E_{A, n+1}^{\prime}\right]
\end{array}\right) \\
\mathbf{F}_{B} & =\left(\begin{array}{c}
E_{B, n}-\mathcal{B}\left[E_{B, n}\right] \\
E_{B, n+1}^{\prime}-\mathcal{B}\left[E_{B, n+1}^{\prime}\right]
\end{array}\right) \\
\mathbf{G}_{A} & =\left(\begin{array}{c}
\mathcal{C}\left[E_{A, n}\right] \\
\mathcal{C}\left[E_{A, n+1}^{\prime}\right]
\end{array}\right) \\
\mathbf{G}_{B} & =\left(\begin{array}{c}
\mathcal{D}\left[E_{B, n}\right] \\
\mathcal{D}\left[E_{B, n+1}^{\prime}\right]
\end{array}\right),
\end{aligned}
$$

which allows us to write the errors in the following form

$$
\begin{aligned}
& \Delta_{A, n+1}=\mathbf{F}_{A}^{T} \mathbf{X}_{A}-\mathbf{G}_{B}^{T} \mathbf{X}_{B}-E_{t, A} \\
& \Delta_{B, n+1}=\mathbf{F}_{B}^{T} \mathbf{X}_{B}-\mathbf{G}_{A}^{T} \mathbf{X}_{A}-E_{t, B} .
\end{aligned}
$$

This time we require both errors to be minimum when averaged over the transverse coordinates. In other words, we have to search for the set of coefficients $a, b, c$, and $d$ that simultaneously minimize the following merit functions

$$
\begin{aligned}
& e_{A}=\sum \Delta_{A, n+1}^{*} \Delta_{A, n+1} \\
& e_{B}=\sum \Delta_{B, n+1}^{*} \Delta_{B, n+1}
\end{aligned}
$$

where the summation is, as before, over all the samples of the window used. The optimal solution is found when the derivative of the above equations with respect to the coefficients of the guess field is zero. To be more precise, we need to set the derivative of $e_{A}$ with respect to $\mathbf{X}_{A}^{+}$to zero, assuming $\mathbf{X}_{B}$ is already known. In the same way we must set the derivative of $e_{B}$ with respect to $\mathbf{X}_{B}^{+}$to zero, assuming $\mathbf{X}_{A}$ to be known. The simultaneous solution of both the resulting equations will yield the desired optimal coefficients. After some algebra, the following equations are found:

$$
\left(\sum \mathbf{F}_{A}^{+} \mathbf{F}_{A}\right) \mathbf{x}_{A}-\left(\sum \mathbf{F}_{A}^{+} \mathbf{G}_{B}\right) \mathbf{x}_{B}=\sum \mathbf{F}_{A}^{+} E_{t, A}
$$

$$
\left(\sum \mathbf{F}_{B}^{+} \mathbf{F}_{B}\right) \mathbf{X}_{B}-\left(\sum \mathbf{F}_{B}^{+} \mathbf{G}_{A}\right) \mathbf{X}_{A}=\sum \mathbf{F}_{B}^{+} E_{t, B}
$$

From this result, it is straightforward to extend to the more general case of an arbitrary number of cavities. To the $i$ th cavity, there is a corresponding equation contained on the left hand side the term $\left(\sum \mathbf{F}_{i}^{+} \mathbf{F}_{i}\right) \mathbf{X}_{i}$ and all the terms $-\left(\sum \mathbf{F}_{i}^{+} \mathbf{G}_{j}\right) \mathbf{X}_{\mathbf{j}}$ with $j \neq i$. The right hand side will contain the term $\sum \mathbf{F}_{\mathbf{i}}^{+} E_{t, i}$, which corresponds to the input field that enters directly into the cavity, if any. 


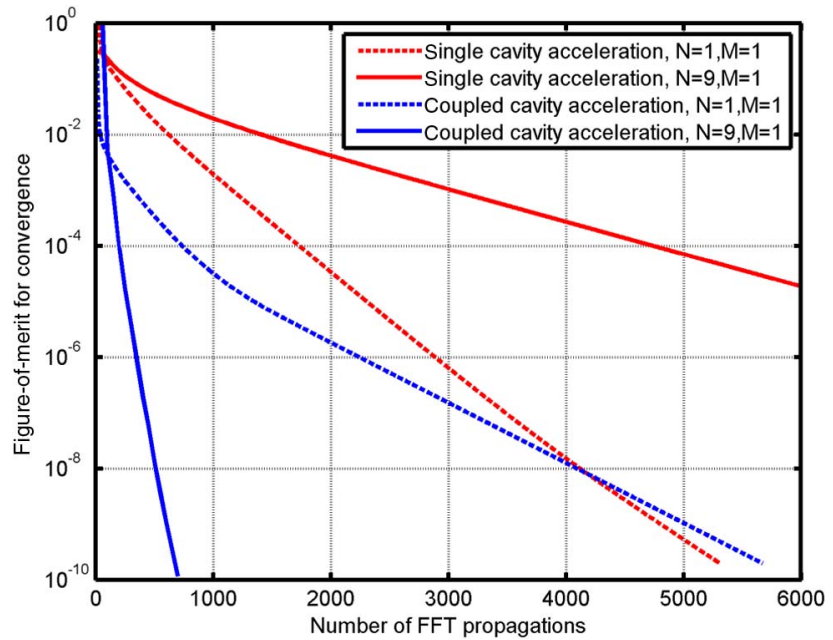

Fig. 11. Comparison of convergence for a double cavity using single cavity acceleration (red lines) and coupled cavity acceleration (blue lines). For clarity, the figure-of-merit has been shown only for the low power cavity. First cavity is concave-convex of length $11.953 \mathrm{~m}$ and second cavity is concave-concave of length $3000 \mathrm{~m}$. Radius of curvature values for input, middle, and end mirrors were $1431 \mathrm{~m}, 1420 \mathrm{~m}$, and $1683 \mathrm{~m}$, respectively. Transmission values of input, middle, and end mirrors were $5 \%, 1.4 \%$, and $1 \mathrm{ppm}$, respectively. Mirror diameters were $350 \mathrm{~mm}$. Input beam was mode-matched to the first cavity.

We will give, as an example, the simulation of a double cavity similar to those found in GW interferometers. In Fig. 11, we present the cavity parameters and results of convergence.

Using realistic mirror maps with rms of $0.5 \mathrm{~nm}$, the power in the cavities for $1 \mathrm{~W}$ at input was $50 \mathrm{~W}$ and $14 \mathrm{~kW}$ for the first and second cavity, respectively. Because of the much higher power in the second cavity, previous simulations have used the approach of accelerating the second cavity while continuing to use standard convergence on the first cavity. We refer to this technique as single cavity acceleration. This results in the convergence given by the dashed red line in Fig. 11. When using the coupled cavity acceleration described in this section, we obtained the blue dashed line. We can see, in this example, that there was no advantage to using coupled cavity acceleration. The reason for this is the unfavorable combination of mirror aberrations and low power in the first cavity. However, we gain considerably by implementing the smoothed acceleration. We can see that the use of smoothed acceleration for the single cavity acceleration (solid red curve) is totally ineffective. This is because the convergence of the second cavity, which is accelerated, is limited by the first cavity that uses standard convergence. The coupled cavity acceleration was, however, able to profit from smoothed acceleration (solid blue curve), and, in this example, it converged more than seven times faster than the other methods.

As we have mentioned previously, the coupled cavity scheme is of particular interest when there is a large amount of power in all cavities that are coupled. An example configuration that is therefore well adapted to this technique is a dispersively coupled optomechanical system [14]. This configuration consists of placing a membrane inside a cavity with a finesse of the order of one million. The membrane typically has a reflectivity of the order of a few percent. The ensemble may therefore be treated as two coupled cavities. In Fig. 12, we present the cavity parameters and results of convergence.

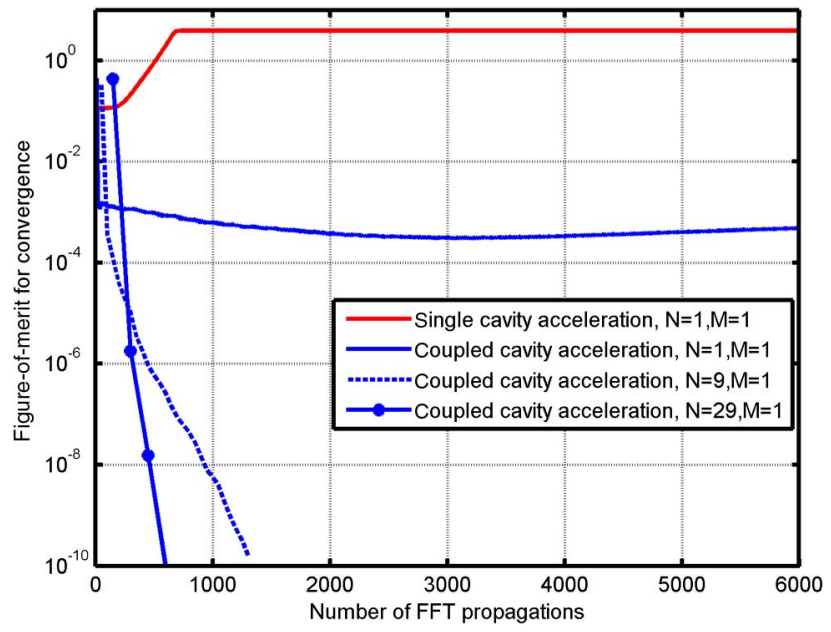

Fig. 12. Comparison of convergence for a double cavity using single cavity acceleration (red line) and coupled cavity acceleration (blue lines). For clarity, the figure-of-merit has been shown only for the low power cavity. First cavity is concave-flat of length $59.5 \mathrm{~mm}$ and second cavity is flat-concave of length $59.5 \mathrm{~mm}$. Radius of curvature for the input and end mirrors was $200 \mathrm{~mm}$. Transmission values of input, middle, and end mirrors were $1 \mathrm{ppm}, 96 \%$, and $1 \mathrm{ppm}$, respectively. Mirror diameters were $1.2 \mathrm{~mm}$. Input beam was mode-matched to first cavity.

Using realistic mirror maps with rms of $0.05 \mathrm{~nm}$, the power in the cavities for $1 \mathrm{~W}$ at input was 287 and $430 \mathrm{~kW}$ for the first and second cavity, respectively. Both the single and coupled cavity acceleration were unable to converge within a reasonable time; the extremely high finesse of the cavity appears to generate an unstable state that, at the time of writing, could not be explained. However, the dashed and dotted curves in Fig. 12 show that, by using smoothed acceleration with a sufficiently large value for $\mathrm{N}$, the stability was regained resulting in an extremely fast convergence.

\section{CONCLUSION}

In this paper, we have presented a complete method for accelerated convergence of FFT simulations. Our approach is a direct extension of the work done by Saha [7]. We have complemented his work by studying in more detail the intrinsic limitations of such a scheme. We found that there were a number of important factors that could hinder the performance of the accelerated convergence such as the working precision, the proportion of HOMs in the cavity, and FFT aliasing. We presented methods for tackling these issues effectively. Notably, we presented the concept of smoothed and averaged acceleration, which provides a consistent speed improvement over classic accelerated convergence. The product of this work was a convergence scheme that was much more reliable and predictable.

We went on to present a method that extends Saha's single cavity accelerated convergence to the case of double and multiple cavities. We demonstrated that there was considerable benefit in using such a scheme for a GW-interferometer type optical configuration. However, we also demonstrated that this scheme is absolutely essential in the case of high finesse coupled cavities whereby there is a similar power stored in each cavity. Our numerical simulations showed that for such a challenging simulation, the smoothed acceleration method not only allowed faster convergence but also acted to 
reduce numerical instabilities resulting in a more robust method.

The increased speed, versatility, and robustness of the accelerated convergence scheme presented in this paper makes this an important tool for FFT simulation in many fields of laser physics.

\section{ACKNOWLEDGMENTS}

The research activity of one of the authors (GV) was partially supported by Regione Toscana (Italy) through the program POR CreO FSE 2007-2013 of the European Community, within the project no. 18113 (ISAV). LIGO was constructed by the California Institute of Technology and Massachusetts Institute of Technology with funding from the United States National Science Foundation under grant PHY-0757058.

\section{REFERENCES}

1. A. E. Siegman, Lasers (University Science, 1986).

2. A. Freise, G. Heinzel, H. Luck, R. Schilling, B. Willke, and K. Danzmann, "Frequency-domain interferometer simulation with higher-order spatial modes," Class. Quantum Grav. 21, S1067-S1074 (2004).

3. G. Vajente, "Fast modal simulation of paraxial optical systems: the MIST open source toolbox," Class. Quantum Grav. 30, 075014 (2013).

4. W. Winkler, R. Schilling, K. Danzmann, J. Mizuno, A. Rüdiger, and K. A. Strain, "Light scattering described in the mode picture," Appl. Opt. 33, 7547-7550 (1994).

5. J.-Y. Vinet, P. Hello, C. N. Man, and A. Brillet, "A high accuracy method for the simulation of non-ideal optical cavities," J. Phys. I 2, 1287-1303 (1992).
6. C. Bradaschia, R. Del Fabbro, A. Di Virgilio, A. Giazotto, H. Kautzky, V. Montelatici, D. Passuello, A. Brillet, O. Cregut, P. Hello, C. N. Man, P. T. Manh, A. Marraud, D. Shoemaker J. Y. Vinet, F. Barone, L. Di Fiore, L. Milano, G. Russo, J. M. Aguirregabiria, H. Bel, J. P. Duruisseau, G. Le Denmat, Ph. Tourrenc, M. Capozzi, M. Longo, M. Lops, I. Pinto, G. Rotoli, T. Damour, S. Bonazzola, J. A. Marck, Y. Gourghoulon, L. E. Holloway, F. Fuligni, V. Iafolla, and G. Natale, "The VIRGO Project: a wide band antenna for gravitational wave detection," Nucl. Instrum. Methods Phys. Res. A Accel. Spectrom. Detect. Assoc. Equip. 289, 518-525 (1990).

7. P. Saha, "Fast estimation of transverse fields in high-finesse optical cavities,” J. Opt. Soc. Am. A 14, 2195-2202 (1997).

8. B. Bochner and Y. Hefetz, "Grid-based simulation program for gravitational wave interferometers with realistically imperfect optics," Phys. Rev. D 68, 082001 (2003).

9. B. Bochner, "Modelling the performance of interferometric gravitational-wave detectors with realistically imperfect optics," Ph.D. dissertation (Massachussets Institute of Technology, 1998).

10. MATLAB, The MathWorks, Inc., Natick, Massachusetts, USA.

11. R. W. P. Drever, J. L. Hall, F. V. Kowalski, J. Hough, G. M. Ford, A. J. Munley, and H. Ward, "Laser phase and frequency stabilization using an optical resonator," Appl. Phys. B 31, 97-105 (1983).

12. Multiprecision Computing Toolbox, Advanpix, Yokohama, Japan.

13. K. Matsushima and T. Shimobaba, "Band-limited angular spectrum method for numerical simulation of free-space propagation in far and near fields," Opt. Express 17, 19662-19673 (2009)

14. A. M. Jayich, J. C. Sankey, B. M. Zwickl, C. Yang, J. D. Thompson, S. M. Girvin, A. A. Clerk, F. Marquardt, and J. G. E. Harris, "Dispersive optomechanics: a membrane inside a cavity," New J. Phys. 10, 095008 (2008). 